TRANSACTIONS OF THE

AMERICAN MATHEMATICAL SOCIETY

Volume 364, Number 10, October 2012, Pages 5035-5055

S 0002-9947(2012)05661-3

Article electronically published on April 30, 2012

\title{
HIDDEN SUBSPACE ALGORITHM IN WHITE NOISE ANALYSIS
}

\author{
JEREMY J. BECNEL
}

\begin{abstract}
In 2003, Professors Lomonaco and Kauffman developed an algorithm to find a hidden subspace of a functional. The algorithm was developed in the spirit of Feynman path integrals but lacked mathematical rigor. In this paper we make use of the framework of the White Noise Distribution Theory to supply the appropriate mathematical machinery to make the algorithm rigorous. In the process we construct a Gaussian measure for affine subspaces of a Hilbert space and develop a decomposition theorem for these measures.
\end{abstract}

\section{INTRODUCTION}

The field of quantum computing gained much fame with the development of Shor's quantum factoring algorithm in 1995. Using the fundamental ideas from the factoring algorithm and a few new concepts, many new quantum algorithms have been constructed. At first most quantum algorithms were designed to run on a finite dimensional state space. However, many researchers have turned their attention to developing quantum algorithms which operate on various other state spaces.

In [9], Professors Lomonaco and Kauffman developed a quantum hidden subspace algorithm. This algorithm takes a function $\phi$ on a Hilbert space and attempts to find a subspace $V$ such that $\phi(x+v)=\phi(x)$ for all $v \in V$. This algorithm is presented in 9 at a very formal level. It is based on being able to do computation in the spirit of Feynman path integrals.

In this work we will make use of the tools of the White Noise Distribution Theorem to give meaning to many of the constructs used in the algorithm.

We begin in Section 2 by presenting the Quantum Hidden Subspace algorithm developed in 9]. We then lay out the fundamentals of white noise analysis in Sections 3 and [4. In Sections 5 and 6 we develop an infinite dimensional Gaussian measure for affine subspaces of a Hilbert space and prove some results involving these measures. Finally, in Section 7 we connect these two subjects in order to provide rigor to the Quantum Hidden Subspace Algorithm.

\section{Hidden Subspace Algorithm}

Let $H$ be a Hilbert space with inner product $\langle\cdot, \cdot\rangle$. Further, suppose we have a functional $\phi: H \rightarrow \mathbb{R}^{n}$ with a hidden subspace $V \subset H$ such that

$$
\phi(x+v)=\phi(x) \quad \text { for all } v \in V .
$$

Received by the editors July 21, 2010.

2010 Mathematics Subject Classification. Primary 60H40; Secondary 81P68.

Key words and phrases. White noise analysis, quantum computing, hidden subspace algorithm. This research was supported by Stephen F. Austin Faculty Research Grant. 
The Hidden Subspace Algorithm attempts to find the hidden subspace $V$. The algorithm we present is based on the work of Lomonaco and Kauffman [9].

Remark 2.1. As the authors admit, the algorithm is "highly speculative". It is based on functional integrals and uses a Lebesgue type measure, $D x$ on $H$, which does not exist. The algorithm calculations are all done at a very formal level.

2.1. Rigged Hilbert spaces. In order to develop such an algorithm, we will have to momentarily abandon the structure of a Hilbert space and work with a rigged Hilbert space, also known as a Gel'fand triple. In particular, we will use the rigged Hilbert space $\left(\mathcal{S}\left(\mathbb{R}^{n}\right), L^{2}\left(\mathbb{R}^{n}\right), \mathcal{S}^{\prime}\left(\mathbb{R}^{n}\right)\right)$, where $\mathcal{S}\left(\mathbb{R}^{n}\right)$ is the space of test functions and $\mathcal{S}^{\prime}\left(\mathbb{R}^{n}\right)$ is the space of generalized functions or distributions.

The most common generalized function, and the one we will be most concerned with, is the Dirac delta function, $\delta(x)$, which has the following effect

$$
\int_{\mathbb{R}^{n}} \delta(x) f(x) d x=f(0)
$$

for suitable $f$ (bounded, continuous functions, for instance).

We will use the notation $H_{\mathbb{R}^{n}}$ for this rigged Hilbert space. This space has the orthonormal basis

$$
\left\{|x\rangle ; x \in \mathbb{R}^{n}\right\},
$$

where by orthonormal we mean that

$$
\langle x \mid y\rangle=\delta(x-y) .
$$

For a function $f$ in $L^{2}\left(\mathbb{R}^{n}\right)$ or $\mathcal{S}^{\prime}\left(\mathbb{R}^{n}\right)$, we have the element in $H_{\mathbb{R}^{n}}$ given by the formal integral

$$
\int_{\mathbb{R}^{n}} d x f(x)|x\rangle
$$

For a particular $x_{0}$, we define

$$
\left|x_{0}\right\rangle=\int_{\mathbb{R}^{n}} d x\left\langle x_{0} \mid x\right\rangle|x\rangle=\int_{\mathbb{R}^{n}} d x \delta\left(x-x_{0}\right)|x\rangle,
$$

which gives us

$$
\left\langle x_{0} \mid y_{0}\right\rangle=\delta\left(x_{0}-y_{0}\right) .
$$

In addition to $H_{\mathbb{R}^{n}}$ we will need another rigged Hilbert space. We denote it by $\mathbb{H}$. It is the rigged Hilbert space with orthonormal basis

$$
\{|x\rangle ; x \in H\},
$$

where we have the bracket product defined as

$$
\langle x \mid y\rangle=\delta(x-y) .
$$

2.2. Black box. For the algorithm we will need an element in $\mathbb{H}$ to hold arguments of the function $\phi$ and another element in $H_{\mathbb{R}^{n}}$ to hold the values of $\phi$. We will also make use of a black box

$$
U_{\phi}: \mathbb{H} \otimes H_{\mathbb{R}^{n}} \rightarrow \mathbb{H} \otimes H_{\mathbb{R}^{n}},
$$

which performs the operation

$$
U_{\phi}|x\rangle|z\rangle=|x\rangle|z+\phi(x)\rangle .
$$


2.3. The algorithm. We now outline the algorithm. In the following, we use the notation $\mathcal{F}$ for a formal Fourier transformation.

Remark 2.2. The algorithm relies heavily on the identity,

$$
\int_{V^{\perp}} \delta(y-u) D u=\int_{V} e^{-2 \pi i\langle v, y\rangle} D v .
$$

This identity is not obvious and is developed in [1] for the white noise setting.

\section{Algorithm: Hidden subspace.}

Inputs: the elements $|0\rangle \in \mathbb{H}$ and $|0\rangle \in H_{\mathbb{R}^{n}}$; a black box $U_{\phi}$ which has the effect $U_{\phi}|x\rangle|z\rangle=|x\rangle|z+\phi(x)\rangle$;

Outputs: a vector $u \in V^{\perp}$.

\section{Procedure.}

1. Start in the initial state: $|0\rangle|0\rangle \in \mathbb{H} \otimes H_{\mathbb{R}^{n}}$.

2. Apply $\mathcal{F}^{-1} \otimes I$ to create the superposition

$$
\rightarrow \int_{H} D x e^{2 \pi i\langle x, 0\rangle}|x\rangle|0\rangle=\int_{H} D x|x\rangle|0\rangle .
$$

3. Apply the black box $U_{\phi}$ :

$$
\rightarrow \int_{H} D x|x\rangle|\phi(x)\rangle
$$

4. Apply $\mathcal{F} \otimes I:$

$$
\begin{array}{rl}
\rightarrow \int_{H} D & y \int_{H} D x e^{-2 \pi i\langle x, y\rangle}|y\rangle|\phi(x)\rangle=\int_{H} D y|y\rangle \int_{H} D x e^{-2 \pi i\langle x, y\rangle}|\phi(x)\rangle \\
& =\int_{H} D y|y\rangle \int_{V} D v \int_{v+V^{\perp}} D x e^{-2 \pi i\langle x, y\rangle}|\phi(x)\rangle \quad \text { using } H=\bigcup_{v \in V}\left(v+V^{\perp}\right) \\
& =\int_{H} D y|y\rangle \int_{V} D v \int_{V^{\perp}} D x e^{-2 \pi i\langle x+v, y\rangle}|\phi(x+v)\rangle \quad \text { by change of variables } \\
& =\int_{H} D y|y\rangle \int_{V} D v e^{-2 \pi i\langle v, y\rangle} \int_{V^{\perp}} D x e^{-2 \pi i\langle x, y\rangle}|\phi(x)\rangle \quad \text { since } \phi(x+v)=\phi(x) \\
& =\int_{H} D y|y\rangle \int_{V^{\perp}} D u \delta(y-u) \int_{V^{\perp}} D x e^{-2 \pi i\langle x, y\rangle}|\phi(x)\rangle \quad \text { by equation (2.1) } \\
= & \int_{V^{\perp}} D u|u\rangle \int_{V^{\perp}} D x e^{-2 \pi i\langle x, u\rangle}|\phi(x)\rangle \\
= & \int_{V^{\perp}} D u|u\rangle|\Omega(u)\rangle,
\end{array}
$$

where

$$
|\Omega(u)\rangle=\int_{V^{\perp}} D x e^{-2 \pi i\langle x, u\rangle}|\phi(x)\rangle .
$$

5. Measure the first register with respect to the observable

$$
M=\int_{H} D w|w\rangle\langle w|
$$

to produce a random vector $u \in V^{\perp}$. 
Remark 2.3. In the original algorithm by Lomonaco and Kauffman [9, $H$ was taken to be the space paths of all functions (paths) $x:[0,1] \rightarrow \mathbb{R}^{n}$, which are $L^{2}$ with respect to the inner product

$$
x \cdot y=\int_{0}^{1} d t x(t) y(t),
$$

and the measure $D x$ was taken in line with Feynman path integrals.

This algorithm provides the motivation for the work to follow. We focus on making the notions introduced by this algorithm mathematically rigorous. To do this we will need the mathematical machinery of White Noise Analysis or Infinite Dimensional Distribution Theory. The next section introduces the concepts necessary for working in this subject.

\section{White NOISE SETUP}

We begin by describing the setting under which White Noise Analysis takes place. Because of the absence of the Lebesgue measure in infinite dimensions, we begin by constructing a Gaussian measure. From here we can develop appropriate sets of test functions and distributions.

We work with a real separable Hilbert space $H_{0}$, and a positive Hilbert-Schmidt operator $A$ on $H_{0}$ such that there is orthonormal basis $\left\{e_{n}\right\}_{n=1}^{\infty}$ of eigenvectors of $A$ and eigenvalues $\left\{\lambda_{n}\right\}_{n=1}^{\infty}$ satisfying

(1) $A e_{n}=\lambda_{n} e_{n}$,

(2) $1<\lambda_{1}<\lambda_{2}<\cdots$,

(3) $\sum_{n=1}^{\infty} \lambda_{n}^{-2}<\infty$.

The example to keep in mind is (see [3])

$$
\begin{aligned}
H_{0} & =L^{2}(\mathbb{R}) \\
e_{n} & =(-1)^{n} \frac{1}{\sqrt{n !}}(2 \pi)^{-1 / 4} e^{x^{2} / 4} \frac{d^{n} e^{-x^{2} / 2}}{d x^{n}}, \\
A & =-\frac{d^{2}}{d x^{2}}+\frac{x^{2}}{4}+\frac{1}{2} \quad \text { with eigenvalues } \lambda_{n}=(n+1) .
\end{aligned}
$$

We have the coordinate map

$$
J: H_{0} \mapsto \mathbb{R}^{W}: f \mapsto\left(\left\langle f, e_{n}\right\rangle\right)_{n \in W},
$$

where we use the notation $W=\{1,2, \ldots\}$. Let

$$
F_{0}=J\left(H_{0}\right)=\left\{\left(x_{n}\right)_{n \in W}: \sum_{n \in W} x_{n}^{2}<\infty\right\} .
$$

Now, for each $p \in W$, let

$$
F_{p}=\left\{\left(x_{n}\right)_{n \in W}: \sum_{n \in W} \lambda_{n}^{2 p} x_{n}^{2}<\infty\right\} .
$$

On $F_{p}$ we have the inner product $\langle\cdot, \cdot\rangle_{p}$ given by

$$
\langle a, b\rangle_{p}=\sum_{n \in W} \lambda_{n}^{2 p} a_{n} b_{n}
$$


This makes $F_{p}$ a real Hilbert space, unitarily isomorphic to $L^{2}\left(W, \mu_{p}\right)$, where $\mu_{p}$ is the measure on $W$ specified by $\mu_{p}(\{n\})=\lambda_{n}^{2 p}$. Moreover, we have

$$
F \stackrel{\text { def }}{=} \bigcap_{p \in W} F_{p} \subset \cdots \subset F_{2} \subset F_{1} \subset F_{0}=L^{2}\left(W, \mu_{0}\right),
$$

and each inclusion $F_{p+1} \rightarrow F_{p}$ is Hilbert-Schmidt.

Now we pull all this back to $H_{0}$. First set

$$
H_{p}=J^{-1}\left(F_{p}\right)=\left\{x \in H_{0}: \sum_{n \in W} \lambda_{n}^{2 p}\left|\left\langle x, e_{n}\right\rangle\right|^{2}<\infty\right\} .
$$

It is readily checked that $H_{p}=\left\{x \in H_{0} ;|x|_{p}<\infty\right\}$ where $|x|_{p}=\left|A^{p} x\right|_{0}$ and also $H_{p}=A^{-p}\left(H_{0}\right)$. On $H_{p}$ we have the pull-back inner product $\langle\cdot, \cdot\rangle_{p}$, which works out to

$$
\langle f, g\rangle_{p}=\left\langle A^{p} f, A^{p} g\right\rangle .
$$

Then we have the chain

$$
\mathcal{H} \stackrel{\text { def }}{=} \bigcap_{p \in W} H_{p} \subset \cdots \subset H_{2} \subset H_{1} \subset H_{0},
$$

with each inclusion $H_{p+1} \rightarrow H_{p}$ being Hilbert-Schmidt.

Equip $\mathcal{H}$ with the topology generated by the norms $|\cdot|_{p}$ (i.e., the smallest topology making all inclusions $\mathcal{H} \rightarrow H_{p}$ continuous). Then $\mathcal{H}$ is, more or less by definition, a nuclear space. The vectors $e_{n}$ all lie in $\mathcal{H}$ and the set of all rational-linear combinations of these vectors produces a countable dense subspace of $\mathcal{H}$.

Consider a linear functional on $\mathcal{H}$ which is continuous. Then it must be continuous with respect to some norm $|\cdot|_{p}$. Thus the topological dual $\mathcal{H}^{\prime}$ is the union of the duals $H_{p}^{\prime}$. In fact, we have:

$$
\mathcal{H}^{\prime}=\bigcup_{p \in W} H_{p}^{\prime} \supset \cdots \supset H_{2}^{\prime} \supset H_{1}^{\prime} \supset H_{0}^{\prime} \simeq H_{0},
$$

where in the last step we used the usual Hilbert space isomorphism between $H_{0}$ and its dual $H_{0}^{\prime}$.

Going over to the sequence space, $H_{p}^{\prime}$ corresponds to

$$
F_{-p} \stackrel{\text { def }}{=}\left\{\left(x_{n}\right)_{n \in W}: \sum_{n \in W} \lambda_{n}^{-2 p} x_{n}^{2}<\infty\right\} .
$$

The element $y \in F_{-p}$ corresponds to the linear functional on $F_{p}$ given by

$$
x \mapsto \sum_{n \in W} x_{n} y_{n},
$$

which, by Cauchy-Schwarz, is well defined and does define an element of the dual $F_{p}^{\prime}$ with norm equal to $|y|_{-p}$.

3.1. Gaussian measure in infinite dimensions. Now consider the product space $\mathbb{R}^{W}$, along with the coordinate projection maps

$$
\hat{X}_{j}: \mathbb{R}^{W} \rightarrow \mathbb{R}: x \mapsto x_{j}
$$

for each $j \in W$. Equip $\mathbb{R}^{W}$ with the product $\sigma$-algebra, i.e., the smallest $\sigma$-algebra with respect to which each projection map $\hat{X}_{j}$ is measurable. Kolmogorov's theorem on infinite products of probability measures provides a probability measure $\nu$ on 
the product $\sigma$-algebra such that each function $\hat{X}_{j}$, viewed as a random variable, has standard Gaussian distribution. Thus,

$$
\int_{\mathbb{R}^{W}} e^{i t \hat{X}_{j}} d \nu=e^{-t^{2} / 2}
$$

for $t \in \mathbb{R}$, and every $j \in W$. The measure $\nu$ is the product of the standard Gaussian measure $e^{-x^{2} / 2}(2 \pi)^{-1 / 2} d x$ on each component $\mathbb{R}$ of the product space $\mathbb{R}^{W}$.

Since, for any $p \geq 1$, we have

$$
\int_{\mathbb{R}^{W}} \sum_{j \in W} \lambda_{j}^{-2 p} x_{j}^{2} d \nu(x)=\sum_{j \in W} \lambda_{j}^{-2 p}<\infty,
$$

it follows that $\nu\left(F_{-p}\right)=1$ for all $p \geq 1$. Thus $\nu\left(F^{\prime}\right)=1$.

We can, therefore, transfer the measure $\nu$ back to $\mathcal{H}^{\prime}$, obtaining a probability measure $\mu$ on the $\sigma$-algebra of subsets of $\mathcal{H}^{\prime}$ generated by the maps

$$
\hat{e}_{j}: \mathcal{H}^{\prime} \rightarrow \mathbb{R}: f \mapsto f\left(e_{j}\right),
$$

where $\left\{e_{j}\right\}_{j \in W}$ is the orthonormal basis of $H_{0}$ we started with (note that each $e_{j}$ lies in $\left.\mathcal{H}=\bigcap_{p>0} H_{p}\right)$. This is clearly the $\sigma$-algebra generated by the weak topology on $\mathcal{H}^{\prime}$ (which happens to be equal also to the $\sigma$-algebras generated by the strong/inductive-limit topology [1]).

Specialized to the example $H_{0}=L^{2}(\mathbb{R})$, and $A=-\frac{d^{2}}{d x^{2}}+\frac{x^{2}}{4}+\frac{1}{2}$, we have the standard Gaussian measure on the distribution space $\mathcal{S}^{\prime}(\mathbb{R})$.

The above discussion gives a simple direct description of the measure $\mu$. Its existence is also obtainable by applying the Minlos theorem:

Theorem 3.1 (Minlos theorem). A complex valued function $\phi$ on a nuclear space $\mathcal{H}$ is the characteristic function of a unique probability measure $\nu$ on $\mathcal{H}^{\prime}$, i.e.,

$$
\phi(y)=\int_{\mathcal{H}^{\prime}} e^{i\langle x, y\rangle} d \nu(x)=\int_{\mathcal{H}^{\prime}} e^{i \hat{y}(x)} d \nu(x), \quad y \in \mathcal{H},
$$

if and only if $\phi(0)=1, \phi$ is continuous, and $\phi$ is positive definite.

Note that as in Theorem 3.1 it is common to denote the random variable $\hat{y}$ as $\langle\cdot, y\rangle$.

For a proof of the Minlos theorem, refer to 4. Applying the Minlos theorem to the characteristic function $\phi(y)=e^{-\frac{1}{2}|y|_{0}^{2}}$ gives us the standard Gaussian measure $\mu$ we just constructed.

There is also the useful standard setting of Abstract Wiener Spaces for Gaussian measures introduced by L. Gross (see the account in Kuo [8]).

To summarize, we can state the starting point of much of infinite dimensional distribution theory (white noise analysis): Given a real, separable Hilbert space $H_{0}$ and a positive Hilbert-Schmidt operator $A$ on $H_{0}$, we have constructed a nuclear space $\mathcal{H}$ and a unique probability measure $\mu$ on the Borel $\sigma$-algebra of the dual $\mathcal{H}^{\prime}$ such that there is a linear map

$$
H_{0} \rightarrow L^{2}\left(\mathcal{H}^{\prime}, \mu\right): x \mapsto \hat{x},
$$

satisfying

$$
\int_{\mathcal{H}^{\prime}} e^{i t \hat{x}} d \mu=e^{-t^{2}|x|_{0}^{2} / 2}
$$

for every real $t$ and $x \in H_{0}$. This Gaussian measure $\mu$ is often called the white noise measure and forms the background measure for White Noise Distribution Theory. 


\section{White Noise Distribution Theory}

We can now develop the ideas of the preceding section further to construct a space of test functions over the dual space $\mathcal{H}^{\prime}$, where $\mathcal{H}$ is the nuclear space related to a real separable Hilbert space $H_{0}$ as in the discussion in Section 3 . We will use the notation, and in particular the spaces $H_{p}$, from Section 3 .

The symmetric Fock space $\mathcal{F}_{s}(V)$ over a Hilbert space $V$ is the subspace of symmetric tensors in the completion of the tensor algebra $T(V)$ under the inner product given by

$$
\langle a, b\rangle_{T(V)}=\sum_{n=0}^{\infty} n !\left\langle a_{n}, b_{n}\right\rangle_{V^{\otimes n}},
$$

where $a=\left\{a_{n}\right\}_{n \geq 0}, b=\left\{b_{n}\right\}_{n \geq 0}$ are elements of $T(V)$ with $a_{n}, b_{n}$ in the tensor power $V^{\otimes n}$. Then we have

$$
\mathcal{F}_{s}(\mathcal{H}) \stackrel{\text { def }}{=} \bigcap_{p \geq 0} \mathcal{F}_{s}\left(H_{p}\right) \subset \cdots \subset \mathcal{F}_{s}\left(H_{2}\right) \subset \mathcal{F}_{s}\left(H_{1}\right) \subset \mathcal{F}_{s}\left(H_{0}\right) .
$$

Thus, the pair $\mathcal{H} \subset H_{0}$ gives rise to a corresponding pair by taking symmetric Fock spaces:

$$
\mathcal{F}_{s}(\mathcal{H}) \subset \mathcal{F}_{s}\left(H_{0}\right) .
$$

4.1. Wiener-Itô isomorphism. In infinite dimensions the role of Lebesgue measure is played by Gaussian measure $\mu$. There is a standard unitary isomorphism, the Wiener-Itô isomorphism or wave-particle duality map, which identifies the complexified Fock space $\mathcal{F}_{s}\left(H_{0}\right)_{c}$ with $L^{2}\left(\mathcal{H}^{\prime}, \mu\right)$. This is accomplished by using the multiple Wiener-Itô integral

$$
I_{n}: H_{0, c}^{\widehat{\otimes} n} \rightarrow L^{2}\left(\mathcal{H}^{\prime}, \mu\right) .
$$

Theorem 4.1 (Wiener-Itô). Each $\phi \in L^{2}\left(\mathcal{H}^{\prime}, \mu\right)$ can be uniquely expressed as

$$
\phi=\sum_{n=0}^{\infty} I_{n}\left(f_{n}\right),
$$

where $\left(f_{n}\right)_{n=0}^{\infty} \in \mathcal{F}_{s}\left(H_{0}\right)_{c}$. Conversely, for any $\left(f_{n}\right)_{n=0}^{\infty} \in \mathcal{F}_{s}\left(H_{0}\right)_{c}$, we have that (4.4) defines a function in $L^{2}\left(\mathcal{H}^{\prime}, \mu\right)$. Moreover,

$$
\|\phi\|_{0}^{2}=\sum_{n=0}^{\infty} n !\left|f_{n}\right|_{0}^{2} .
$$

For a proof refer to Obata's book [10]. The map which identifies $\phi \in L^{2}\left(\mathcal{H}^{\prime}, \mu\right)$ with its corresponding $\left(f_{n}\right)_{n=0}^{\infty} \in \mathcal{F}_{s}\left(H_{0}\right)_{c}$ is called the Wiener-Itô isomorphism and is uniquely specified by

$$
I: \mathcal{F}_{s}\left(H_{0}\right)_{c} \rightarrow L^{2}\left(\mathcal{H}^{\prime}, \mu\right): \operatorname{Exp}(x) \mapsto e^{\hat{x}-\frac{1}{2}|x|_{0}^{2}},
$$

where $x \in \mathcal{H}$ and

$$
\operatorname{Exp}(x)=\sum_{n=0}^{\infty} \frac{1}{n !} x^{\otimes n} .
$$

Indeed, it is readily checked that $I$ preserves inner products (the inner product is as described in (4.1)). Using $I$, for each $\mathcal{F}_{s}\left(H_{p}\right)$ with $p \geq 0$, we have the corresponding 
space $[H]_{p} \subset L^{2}\left(\mathcal{H}^{\prime}, \mu\right)$ with the norm $\|\cdot\|_{p}$ induced by the norm on the space $\mathcal{F}_{s}\left(H_{p}\right)_{c}$. The chain of spaces (4.2) can be transferred into a chain of function spaces:

$$
[\mathcal{H}]=\bigcap_{p \geq 0}[H]_{p} \subset \cdots \subset[H]_{2} \subset[H]_{1} \subset[H]_{0}=L^{2}\left(\mathcal{H}^{\prime}, \mu\right) .
$$

Observe that $[\mathcal{H}]$ is a nuclear space with topology induced by the norms $\left\{\|\cdot\|_{p} ; p=\right.$ $0,1,2, \ldots\}$. Thus, starting with the pair $\mathcal{H} \subset H_{0}$, one obtains a corresponding pair $[\mathcal{H}] \subset L^{2}\left(\mathcal{H}^{\prime}, \mu\right)$.

As before, the identification of $H_{0}^{\prime}$ with $H_{0}$ leads to a complete chain

$$
\mathcal{H}=\bigcap_{p \geq 0} H_{p} \subset \cdots \subset H_{1} \subset H_{0} \simeq H_{-0} \subset H_{-1} \subset \cdots \subset \bigcup_{p \geq 0} H_{-p}=\mathcal{H}^{\prime} .
$$

In the same way, we have a chain for the 'second quantized' spaces $\mathcal{F}_{s}\left(H_{q}\right)_{c} \simeq[H]_{q}$. The unitary isomorphism $I$ extends to unitary isomorphisms

$$
I: \mathcal{F}_{s}\left(H_{-p}\right)_{c} \rightarrow[H]_{-p} \stackrel{\text { def }}{=}[H]_{p}^{\prime} \subset[\mathcal{H}]^{\prime},
$$

for all $p \geq 0$. In more detail, for $a \in \mathcal{F}_{s}\left(H_{-p}\right)_{c}$ the distribution $I(a)$ is specified by

$$
\langle\langle I(a), \phi\rangle\rangle=\left\langle\left\langle a, I^{-1}(\phi)\right\rangle\right\rangle,
$$

for all $\phi \in[\mathcal{H}]$. On the right side here we have the pairing of $\mathcal{F}_{s}\left(H_{-p}\right)_{c}$ and $\mathcal{F}_{s}\left(H_{p}\right)_{c}$ induced by the duality pairing of $H_{-p}$ and $H_{p}$; in particular, the pairings above are complex bilinear (not sesquilinear).

4.1.1. Wiener-Itô expansion. The expression in (4.4) is called the Wiener-Itô expansion for $\phi$. This type of representation can be extended to functions $\Phi$ in $[\mathcal{H}]^{\prime}$.

Theorem 4.2. Given $\Phi \in[H]_{-p}$, there exists a unique element $\left(F_{n}\right)_{n=0}^{\infty} \in \mathcal{F}_{s}\left(H_{p}^{\prime}\right)_{c}$ such that

$$
\langle\langle\Phi, \phi\rangle\rangle=\sum_{n=0}^{\infty} n !\left\langle F_{n}, f_{n}\right\rangle \quad \text { for all } \phi \in[H]_{p},
$$

where $\phi=\sum_{n=0}^{\infty} I_{n}\left(f_{n}\right)$. Conversely, given a sequence $\left(F_{n}\right)_{n=0}^{\infty} \in \mathcal{F}_{s}\left(H_{p}^{\prime}\right)_{c}$, we can define $\Phi \in[H]_{-p}$ by (4.11). Moreover, we have that

$$
\|\Phi\|_{-p}^{2}=\sum_{n=0}^{\infty} n !\left|F_{n}\right|_{-p}^{2}
$$

For a proof see [7, 10. Using the previous theorem, we can adopt notation for the Wiener-Itô expansion of $\Phi \in[\mathcal{H}]^{\prime}$ as follows:

$$
\Phi=\sum_{n=0}^{\infty} I_{n}\left(F_{n}\right) .
$$

4.2. Properties of test functions. The following theorem summarizes the properties of $[\mathcal{H}]$ which are commonly used. The results here are standard (see, for instance, the monograph [7 by Kuo), and we compile them here for ease of reference. 
Theorem 4.3. Every function in $[\mathcal{H}]$ is $\mu$-almost-everywhere equal to a unique continuous function on $\mathcal{H}^{\prime}$. Moreover, working with these continuous versions,

(1) $[\mathcal{H}]$ is an algebra under pointwise operations;

(2) pointwise addition and multiplication are continuous operations $[\mathcal{H}] \times[\mathcal{H}] \rightarrow$ $[\mathcal{H}]$

(3) for any $\phi \in \mathcal{H}^{\prime}$, the evaluation map

$$
\delta_{\phi}:[\mathcal{H}] \rightarrow \mathbb{R}: F \mapsto F(\phi)
$$

is continuous;

(4) the exponentials $e^{i \hat{x}-\frac{1}{2}|x|_{0}^{2}}$, with $x$ running over $\mathcal{H}$, span a dense subspace of $[\mathcal{H}]$.

A complete characterization of the space $[\mathcal{H}]$ was obtained by Y. J. Lee (see the account in Kuo [7, page 89]).

4.3. The Segal-Bargmann transform. An important tool for studying test functions and distributions in the white noise setting is the Segal-Bargmann transform. The Segal-Bargmann transform takes a function $F \in L^{2}\left(\mathcal{H}^{\prime}, \mu\right)$ to the function $S F$ on the complexified space $\mathcal{H}_{c}$ given by

$$
S F(z)=\int_{\mathcal{H}^{\prime}} e^{\tilde{z}-\langle z, z\rangle^{2} / 2} F d \mu, \quad z \in \mathcal{H}_{c}
$$

with notation as follows. If $z=a+i b$, with $a, b \in \mathcal{H}$, then

$$
\tilde{z}(x) \stackrel{\text { def }}{=}\langle z, x\rangle=\langle a, x\rangle+i\langle b, x\rangle, \quad \text { for } x \in \mathcal{H}^{\prime}
$$

and again, the pairing $\langle z, w\rangle$ for $z, w \in \mathcal{H}_{c}$ is complex bilinear (not sesquilinear).

Let $\mu_{c}$ be the Gaussian measure $\mathcal{H}_{c}^{\prime}$ specified by the requirement that

$$
\int_{\mathcal{H}_{c}^{\prime}} e^{a x+b y} d \mu_{c}(x+i y)=e^{\left(a^{2}+b^{2}\right) / 4}
$$

for every $a, b \in \mathcal{H}$. For convenience, let us introduce the renormalized exponential function $c_{w}=e^{\tilde{w}-\langle w, w\rangle^{2} / 2} \in L^{2}\left(\mathcal{H}^{\prime}, \mu\right)$ for all $w \in \mathcal{H}_{c}$. It is readily checked that for any $w \in \mathcal{H}_{c}$

$$
\left[S c_{w}\right](z)=e^{\langle w, z\rangle}, \quad \text { for all } z \in \mathcal{H}_{c} .
$$

Thus we may take $S c_{w}$ as a function on $\mathcal{H}_{c}^{\prime}$ given by $S c_{w}=e^{\tilde{w}}$ where now $\tilde{w}$ is a function on $\mathcal{H}_{c}^{\prime}$ in the natural way. Then $S c_{w} \in L^{2}\left(\mathcal{H}_{c}^{\prime}, \mu_{c}\right)$ and one has

$$
\left\langle S c_{w}, S c_{u}\right\rangle_{L^{2}\left(\mu_{c}\right)}=\left\langle c_{w}, c_{u}\right\rangle_{L^{2}(\mu)}=e^{\langle w, \bar{u}\rangle} .
$$

This shows that $S$ provides an isometry from the linear span of the renormalized exponentials $c_{w}$ in $L^{2}\left(\mathcal{H}^{\prime}, \mu\right)$ onto the linear span of the complex exponentials $e^{\tilde{w}}$ in $L^{2}\left(\mathcal{H}_{c}^{\prime}, \mu_{c}\right)$. Passing to the closure, one obtains the Segal-Bargmann unitary isomorphism

$$
S: L^{2}\left(\mathcal{H}^{\prime}, \mu\right) \rightarrow \operatorname{Hol}^{2}\left(\mathcal{H}_{c}^{\prime}, \mu_{c}\right)
$$

where $\operatorname{Hol}^{2}\left(\mathcal{H}_{c}^{\prime}, \mu_{c}\right)$ is the closed linear span of the complex exponential functions $e^{\tilde{w}}$ in $L^{2}\left(\mathcal{H}_{c}^{\prime}, \mu_{c}\right)$.

An explicit expression for $S F(z)$ is suggested by (4.13). For any $\phi \in[\mathcal{H}]$ and $z \in \mathcal{H}_{c}^{\prime}$, we have

$$
(S \phi)(z)=\langle\langle I(\operatorname{Exp}(z)), \phi\rangle\rangle
$$


where the right side is the evaluation of the distribution $I(\operatorname{Exp}(z))$ on the test function $\phi$. Indeed it may be readily checked that if $S \phi(z)$ is defined in this way, then $\left[S c_{w}\right](z)=e^{\langle w, z\rangle}$.

In view of (4.17), it natural to extend the Segal-Bargmann transform to distributions: for $\Phi \in[\mathcal{H}]^{\prime}$, define $S \Phi$ to be the function on $\mathcal{H}_{c}$ given by

$$
S \Phi(z) \stackrel{\text { def }}{=}\langle\langle\Phi, I(\operatorname{Exp}(z))\rangle\rangle, \quad z \in \mathcal{H}_{c} .
$$

Using Theorem 4.11 and (4.6), the above can be expressed as

$$
S \Phi(z)=\sum_{n=0}^{\infty}\left\langle F_{n}, z^{\otimes n}\right\rangle,
$$

where the $F_{n}$ 's are from the Wiener-Itô expansion of $\Phi$.

One of the many applications of the the $S$-transform includes its usefulness in characterizing generalized functions in $[\mathcal{H}]^{\prime}$.

Theorem 4.4 (Potthoff-Streit). Suppose a function $F$ on $\mathcal{H}_{c}$ satisfies:

(1) For any $z, w \in \mathcal{H}_{c}$, the function $F(\alpha z+w)$ is an entire function of $\alpha \in \mathbb{C}$.

(2) There exists nonnegative constants $A, p$, and $C$ such that

$$
|F(z)| \leq C e^{A|z|_{p}^{2}} \quad \text { for all } z \in \mathcal{H}_{c} .
$$

Then there is a unique generalized function $\Phi \in[\mathcal{H}]^{\prime}$ such that $F=S \Phi$. Conversely, given such a $\Phi \in[\mathcal{H}]^{\prime}$, then $S \Phi$ satisfies (11) and (2) above.

For a proof see Theorem 8.2 in Kuo's book [7] on page 79.

4.4. $\mathcal{T}$-transform. The $\mathcal{T}$-transform was used by Hida when he introduced the theory of white noise. However, because of the convenient Wiener-Itô decomposition of the renormalized exponentials $e^{\langle\cdot, y\rangle-\langle y, y\rangle / 2}$, the $S$-transform grew in favor. Still, in many instances the $\mathcal{T}$-transform is more useful.

Definition 4.5. The $\mathcal{T}$-transform of a generalized function $\Phi \in[\mathcal{H}]^{\prime}$ is given by

$$
\mathcal{T} \Phi(z)=\left\langle\left\langle\Phi, e^{i\langle\cdot, z\rangle}\right\rangle\right\rangle \quad z \in \mathcal{H}_{c} .
$$

It is easy to check that the $\mathcal{T}$-transform and the $S$-transform are related by

$$
S \Phi(z)=e^{-\langle z, z\rangle / 2} \mathcal{T} \Phi(-i z) \quad \text { and } \quad \mathcal{T} \Phi(z)=e^{-\langle z, z\rangle / 2} S \Phi(i z) \quad z \in \mathcal{H}_{c} .
$$

4.5. Kuo Fourier transform. The $S$-transform can also be used to define a Fourier transform on the space of generalized functions $[\mathcal{H}]^{\prime}$. In the finite dimensional setting the Fourier transform can be written as

$$
\begin{aligned}
\mathcal{F}(f)(y) & =(2 \pi)^{-n / 2} \int_{\mathbb{R}^{n}} e^{-i\langle x, y\rangle+|x|^{2} / 2} f(x) e^{-|x|^{2} / 2} d x \\
& =\int_{\mathbb{R}^{n}} e^{-i\langle x, y\rangle+|x|^{2} / 2} f(x) d \mu_{n}(x),
\end{aligned}
$$

where $\mu_{n}$ is the standard Gaussian measure on $\mathbb{R}^{n}$. We would like to define the Fourier transform of a generalized function $\Phi \in[\mathcal{H}]^{\prime}$ in an analogous manner. Namely, as

$$
\mathcal{F} \Phi(y)=\int_{\mathcal{H}^{\prime}} e^{-i\langle x, y\rangle+|x|^{2} / 2} \Phi(x) d \mu(x)
$$


However, this is a purely symbolic integral. But, if we informally take the $S$ transform of $\mathcal{F} \Phi$, we get

$$
S(\mathcal{F} \Phi)(z)=\int_{\mathcal{H}^{\prime}} e^{-i\langle x, z\rangle} \Phi(x) d \mu(x)=\left\langle\left\langle\Phi, e^{-i\langle\cdot, z\rangle}\right\rangle\right\rangle
$$

for $z \in \mathcal{H}_{c}$. It is easily verified that

$$
\left\langle\left\langle\Phi, e^{-i\langle\cdot, z\rangle}\right\rangle\right\rangle=S(\Phi)(-i z) e^{-\langle z, z\rangle / 2} .
$$

For a generalized function $\Phi \in[\mathcal{H}]^{\prime}$, the function given by (4.19) satisfies the conditions of Theorem 4.4. Therefore there is a unique element $\Psi \in[\mathcal{H}]^{\prime}$ with $S$-transform given by (4.19). With this in mind, we define the Fourier transform of a generalized function as follows:

Definition 4.6. The Kuo Fourier transform of a generalized function $\Phi \in[\mathcal{H}]^{\prime}$ is the unique element $\mathcal{F} \Phi$ in $[\mathcal{H}]^{\prime}$ with $S$-transform given by

$$
S(\mathcal{F} \Phi)(z)=\left\langle\left\langle\Phi, e^{-i\langle\cdot, z\rangle}\right\rangle\right\rangle=S(\Phi)(-i z) e^{-\langle z, z\rangle / 2}, \quad z \in \mathcal{H}_{c} .
$$

It turns out that, defined in this way, the Fourier transform is a continuous linear operator from $[\mathcal{H}]^{\prime}$ into itself. (For a proof, see page 141 in [10] or page 152 in [7].)

4.6. Translation operator. An important operator acting on the space of test functions is the translation operator $T_{y}$ with $y \in \mathcal{H}^{\prime}$.

Definition 4.7. For any $y \in \mathcal{H}^{\prime}$ the translation operator $T_{y}$ on $[\mathcal{H}]$ is defined by

$$
T_{y} \phi(x)=\phi(x+y) .
$$

Since the Gaussian measure is not translation invariant, this operator is more intricate than it first appears. The properties of this operator are summarized in the following theorem.

Theorem 4.8. For any $y \in \mathcal{H}^{\prime}$, the translation operator $T_{y}$ is continuous from $[\mathcal{H}]$ into itself. Moreover, if $y \in H_{p}^{\prime}$ and $q>p$ satisfies $\lambda_{1}^{2(q-p)} \geq 2$, then for all $\phi \in[\mathcal{H}]$,

$$
\left\|T_{y} \phi\right\|_{p} \leq\|\phi\|_{q}\left(1-\frac{2}{\lambda_{1}^{2(q-p)}}\right) \exp \left[\frac{1}{\lambda_{1}^{2(q-p)}}|y|_{-p}^{2}\right]
$$

A proof of this can be found in the book by Kuo [7] (page 138, Theorem 10.21).

\section{Gaussian measure on an affine subspace}

For a subspace $W$ of $\mathbb{R}^{n}$ and $a \in W^{\perp}$, we have the Gaussian measure on $a+W$ given by

$$
\int_{a+W} e^{i\langle x, y\rangle} d \mu_{a+W}(x)=\int_{a+W} e^{i\langle x, y\rangle} e^{-\frac{1}{2}|x-a|^{2}} \frac{d x}{(2 \pi)^{\operatorname{dim} W / 2}}=e^{i\langle a, y\rangle-\frac{1}{2}\left\langle y_{W}, y_{W}\right\rangle},
$$

where $y \in \mathbb{R}^{n}$ and $y_{W}$ is the projection of $y$ onto $W$. We now describe how such a measure can be constructed in a white noise setting. Of course, the Gaussian measure cannot live on $H_{0}$ or $a+W$. However, just as we used the Minlos theorem 
to form the Gaussian measure $\mu$ on $\mathcal{H}^{\prime}$ (which we think of as the Gaussian measure on $H_{0}$ ), we can again use the Minlos theorem to form the Gaussian measure for the affine subspace $a+W$.

5.1. Gaussian measure on $a+V$. For a vector $a \in H_{0}$ and a closed subspace $V$ of $H_{0}$, we can use the Minlos theorem to find that there is a measure $\mu_{a+V}$ on $\mathcal{H}^{\prime}$ with

$$
\int_{\mathcal{H}^{\prime}} e^{i\langle x, y\rangle} d \mu_{a+V}(x)=e^{i\langle a, y\rangle-\frac{1}{2}\left\langle y_{V}, y_{V}\right\rangle}
$$

for any $y \in \mathcal{H}$. Here and throughout this work we use $y_{V}$ to represent the orthogonal projection of $y$ onto a closed subspace $V$. The measure $\mu_{a+V}$ is the Gaussian measure for the affine subspace $a+V$. This measure was originally constructed in [2].

5.2. Supporting sets for $\mu_{a+V}$. For any $w \in H_{0}$ (or $H_{0, c}$ ), we can define the random variable $\hat{w}$ or $\langle\cdot, w\rangle$ as a $\mu_{a+V}$-almost-everywhere defined function in the space $L^{2}\left(\mathcal{H}^{\prime}, \mu_{a+V}\right)$ satisfying

$$
\int_{\mathcal{H}^{\prime}} e^{i t \hat{w}} d \mu_{a+V}=e^{i t\langle a, w\rangle-\frac{t^{2}}{2}\left\langle w_{V}, w_{V}\right\rangle} .
$$

As a consequence we have that $\hat{w}$ is a normal random variable with mean $\langle a, w\rangle$ and variance $\left|w_{V}\right|_{0}^{2}$.

If $V$ happens to be finite dimensional, then the measure $\mu_{a+V}$ reverts to the usual finite dimensional Gaussian measure and lives on $a+V$. However, if $V$ is infinite dimensional, then there is an orthonormal basis $v_{1}, v_{2}, \ldots$ of vectors in $H_{0}$ for $V$. For $V^{\perp}$ we also have a (finite or infinite) orthonormal basis $\left\{w_{k}\right\}$. Using (5.2), we have

$$
\int_{\mathcal{H}^{\prime}} e^{i t \hat{w}_{k}} d \mu_{a+V}=e^{i t\left\langle a, w_{k}\right\rangle} .
$$

Thus $\hat{w}_{k}$ has the Dirac distribution and $\hat{w}_{k}=\left\langle a, w_{k}\right\rangle \mu_{a+V}$-almost-everywhere. Thus $\hat{w}_{k}^{-1}\left(\left\langle a, w_{k}\right\rangle\right)$ is of full measure. Since the $w_{k}$ 's are independent (being orthogonal), we have that

$$
\bigcap_{k} \hat{w}_{k}^{-1}\left(\left\langle a, w_{k}\right\rangle\right)
$$

is of full measure.

Remark 5.1. In particular for the case where $a=0$, we have that

$$
\bigcap_{k} \hat{w}_{k}^{-1}(0)
$$

is of full measure with respect to $\mu_{V}$.

5.3. Hida measure. The Gaussian measure $\mu_{a+V}$ is a special type of measure known as a Hida measure. In this section we define the notion of Hida measure and give an overview of some of its properties.

Definition 5.2. A measure $\nu$ on $\mathcal{H}^{\prime}$ is called a Hida measure if $\phi \in L^{1}(\nu)$ for all $\phi \in[\mathcal{H}]$ and the linear functional

$$
\phi \mapsto \int_{\mathcal{H}^{\prime}} \phi(x) d \nu(x)
$$

is continuous on $[\mathcal{H}]$. 
We say that a generalized function $\Phi \in[\mathcal{H}]^{\prime}$ is induced by a Hida measure $\nu$ if for any $\phi \in[\mathcal{H}]$ we have

$$
\langle\langle\Phi, \phi\rangle\rangle=\int_{\mathcal{H}^{\prime}} \phi(x) d \nu(x) .
$$

The following theorem characterizes those generalized functions which are induced by a Hida measure.

Theorem 5.3. Let $\Phi \in[\mathcal{H}]^{\prime}$. Then the following are equivalent:

(1) For any nonnegative $\phi \in[\mathcal{H}],\langle\langle\Phi, \phi\rangle\rangle \geq 0$.

(2) The function $\mathcal{T}(\Phi)(x)=\left\langle\left\langle\Phi, e^{i\langle\cdot, x\rangle}\right\rangle\right\rangle$ is positive definite on $\mathcal{H}$.

(3) $\Phi$ is induced by a Hida measure.

A proof of this theorem can be found in [7] (page 320, Theorem 15.3).

Corollary 5.4. Let $\nu$ be a finite measure on $\mathcal{H}^{\prime}$ such that for any $x \in \mathcal{H}$,

$$
\left\langle\left\langle\Phi, e^{i\langle\cdot x\rangle}\right\rangle\right\rangle=\int_{\mathcal{H}^{\prime}} e^{i\langle y, x\rangle} d \nu(y)
$$

for some $\Phi \in[\mathcal{H}]^{\prime}$. Then $\Phi$ is induced by $\nu$.

Proof. Since $\left\langle\left\langle\Phi, e^{i\langle\cdot x\rangle}\right\rangle\right\rangle=\int_{\mathcal{H}^{\prime}} e^{i\langle y, x\rangle} d \nu(y)$, it is clear that $\left\langle\left\langle\Phi, e^{i\langle\cdot, x\rangle}\right\rangle\right\rangle$ is positive definite. So we can apply Theorem 5.3 to get a finite measure $m$ which is induced by $\Phi$. Hence for all $\phi \in[\mathcal{H}]$,

$$
\langle\langle\Phi, \phi\rangle\rangle=\int_{\mathcal{H}^{\prime}} \phi d m
$$

Letting $\phi=e^{i\langle\cdot x\rangle}$ in the above equation, we see that the characteristic functions for $m$ and $\nu$ are identical. Therefore $m=\nu$ and we have that $\Phi$ is induced by $\nu$.

Here is another useful theorem which characterizes Hida measures.

Theorem 5.5. A measure $\nu$ on $\mathcal{H}^{\prime}$ is a Hida measure if and only if $\nu$ is supported in $H_{p}^{\prime}$ for some $p \geq 1$ and

$$
\int_{H_{p}^{\prime}} \exp \left[\frac{1}{2}|x|_{-p}^{2}\right] d \nu(x)<\infty .
$$

For a proof of this refer to Kuo's book [7] (page 333, Theorem 15.17).

5.4. Definition of the distribution $\tilde{\delta}_{a+V}$. We now prove that $\mu_{a+V}$ is a Hida measure and develop the corresponding distribution $\tilde{\delta}_{a+V}$ which we think of as the delta function for the affine subspace $a+V\left[2\right.$. Observe the effect of $\mu_{a+V}$ on the renormalized exponential $e^{\langle\cdot, z\rangle-\frac{1}{2}\langle z, z\rangle}$,

$$
\begin{aligned}
\int_{\mathcal{H}^{\prime}} e^{\langle x, z\rangle-\frac{1}{2}\langle z, z\rangle} d \mu_{a+V}(x) & =e^{-\langle z, z\rangle} \int_{\mathcal{H}^{\prime}} e^{\langle x, z\rangle} d \mu_{a+V}(x) \\
& =e^{-\langle z, z\rangle} e^{\langle a, z\rangle+\frac{1}{2}\left\langle z_{V}, z_{V}\right\rangle} \\
& =e^{\langle a, z\rangle-\frac{1}{2}\left\langle z_{V^{\perp}}, z_{V^{\perp}}\right\rangle} .
\end{aligned}
$$


Although $\tilde{\delta}_{a+V}$ was originally developed for $a \in H_{0}$, we could also take $a \in H_{p}^{\prime}$. Let the function $F(z)$ denote the result from the calculations above. That is,

$$
F(z)=e^{\langle a, z\rangle-\frac{1}{2}\left\langle z_{V^{\perp}, z_{V} \perp}\right\rangle} .
$$

We will show that $F(z)$ satisfies properties (1) and (2) of Theorem 4.4 .

For property (1) consider $F(\alpha z+w)$ where $z, w \in \mathcal{H}_{c}$ and $\alpha \in \mathbb{C}$. Then notice that

$$
\begin{aligned}
& F(\alpha z+w)=e^{\langle a, \alpha z+w\rangle-\frac{1}{2}\left\langle\alpha z_{V^{\perp}}+w_{V^{\perp}}, \alpha z_{V^{\perp}}+w_{V^{\perp}}\right\rangle} \\
& =\exp \left[\alpha\langle a, z\rangle+\langle a, w\rangle-\frac{1}{2}\left(\alpha^{2}\left\langle z_{V^{\perp}}, z_{V^{\perp}}\right\rangle+2 \alpha\left\langle z_{V^{\perp}}, w_{V^{\perp}}\right\rangle+\left\langle w_{V^{\perp}}, w_{V^{\perp}}\right\rangle\right)\right] \\
& =e^{-\frac{\alpha^{2}}{2}\left\langle z_{V^{\perp}}, z_{V} \perp\right\rangle} e^{\alpha\left(\langle a, z\rangle-\left\langle z_{V} \perp, w_{V} \perp\right\rangle\right)} e^{\langle a, w\rangle-\frac{1}{2}\left\langle w_{V}, w_{V} \perp\right\rangle},
\end{aligned}
$$

which is an entire function of $\alpha \in \mathbb{C}$.

Now for property (2) of Theorem 4.4 we write $z$ as $z=x+i y$ with $x, y \in \mathcal{H}$ and observe that

$$
\begin{aligned}
|F(z)| & =\left|e^{\langle a, z\rangle-\frac{1}{2}\left\langle z_{V^{\perp}}, z_{V^{\perp}}\right\rangle}\right| \\
& =\left|e^{\langle a, x+i y\rangle-\frac{1}{2}\left\langle x_{V^{\perp}}+i y_{V^{\perp}}, x_{V^{\perp}}+i y_{V^{\perp}}\right\rangle}\right| \\
& =e^{\langle a, x\rangle} e^{-\frac{1}{2}\left|x_{V \perp}\right|_{0}^{2}+\frac{1}{2}\left|y_{V^{\perp}}\right|_{0}^{2}} \\
& \leq e^{\langle a, x\rangle} e^{\frac{1}{2}\left|z_{V^{\perp}}\right|_{0}^{2}} \\
& \leq e^{|a|_{-p}|x|_{p}} e^{\frac{1}{2}|z|_{0}^{2}} \\
& \leq e^{\frac{1}{2}|a|_{-p}^{2}+\frac{1}{2}|z|_{p}^{2}} e^{\frac{1}{2}|z|_{p}^{2}} \quad \text { by Young's inequality } \\
& \leq e^{\frac{1}{2}|a|_{-p}^{2}} e^{\frac{3}{2}|z|_{p}^{2}} .
\end{aligned}
$$

So property (2) of Theorem 4.4 is satisfied.

Therefore by Theorem 4.4 there exist some $\Phi \in[\mathcal{H}]^{\prime}$ such that $S(\Phi)(z)=F(z)$. Then by Corollary 5.4 we have that for $a \in H_{0}\left(\right.$ or $\left.H_{p}^{\prime}\right), \Phi$ is induced by $\mu_{a+V}$. We simply denote this $\Phi$ by $\tilde{\delta}_{a+V}$. This leads us to the following definition:

Definition 5.6. The delta function for the affine subspace $a+V$ is the distribution in $[\mathcal{H}]^{\prime}$ induced by the Hida measure $\mu_{a+V}$. We denote this generalized function by $\tilde{\delta}_{a+V}$.

Thus for any test function $\phi \in[\mathcal{H}]$, we have

$$
\left\langle\left\langle\tilde{\delta}_{a+V}, \phi\right\rangle\right\rangle=\int_{\mathcal{H}^{\prime}} \phi d \mu_{a+V} .
$$

Remark 5.7. The special case where $V=0$ is known as the Kubo-Yokoi delta function [6].

5.5. $\boldsymbol{S}$-transform of $\tilde{\delta}_{a+V}$. Using the definition of the distribution $\tilde{\delta}_{a+V}$ we can directly compute its $S$-transform. By the calculations directly preceding (5.3), we have

$$
S\left(\tilde{\delta}_{a+V}\right)(z)=e^{\langle a, z\rangle-\frac{1}{2}\left\langle z_{V^{\perp}}, z_{V^{\perp}}\right\rangle} \quad \text { for } \quad z \in \mathcal{H}_{c} .
$$


5.6. Fourier transform of $\tilde{\delta}_{a+V}$. Let us now compute the Fourier transform of $\tilde{\delta}_{a+V}$. Note that by definition we have

$$
S\left(\mathcal{F} \tilde{\delta}_{a+V}\right)(z)=\left\langle\left\langle\tilde{\delta}_{a+V}, e^{-i\langle\cdot, z\rangle}\right\rangle\right\rangle=\int_{\mathcal{H}^{\prime}} e^{-i\langle\cdot, z\rangle} d \mu_{a+V}
$$

Therefore using (5.2) we have

$$
S\left(\mathcal{F} \tilde{\delta}_{a+V}\right)(z)=e^{-i\langle a, z\rangle-\frac{1}{2}\left\langle z_{V}, z_{V}\right\rangle} .
$$

The most interesting case occurs when we take $a=0$. Then we have

$$
S\left(\mathcal{F} \tilde{\delta}_{V}\right)(z)=e^{-\frac{1}{2}\left\langle z_{V}, z_{V}\right\rangle} .
$$

We now compare the above to the $S$-transform of $\tilde{\delta}_{V^{\perp}}$ using (5.4)

$$
S\left(\tilde{\delta}_{V^{\perp}}\right)(z)=e^{-\frac{1}{2}\left\langle z_{V}, z_{V}\right\rangle} .
$$

Comparing (5.5) with (5.6) we have that $S\left(\mathcal{F} \tilde{\delta}_{V}\right)(z)=S\left(\tilde{\delta}_{V^{\perp}}\right)(z)$ and therefore $\mathcal{F} \tilde{\delta}_{V}=\tilde{\delta}_{V \perp}$.

Example 5.8. Using the above, we see that for the particular case where $V=0$ we have that $\mathcal{F} \tilde{\delta}_{0}=1$ and $\mathcal{F} 1=\tilde{\delta}_{0}$.

5.7. Wiener-Itô expansion of $\tilde{\delta}_{a+V}$. In this section we develop the WienerItô expansion of $\tilde{\delta}_{a+V}$. This expansion was originally proved in 2. We begin by generalizing the definition of the trace operator and Wick tensor which are typically used in white noise analysis [7.

5.7.1. Subspace trace operator. As usual, let $V$ be a closed subspace of our Hilbert space $H_{0}$.

Definition 5.9. The $V$-trace operator, which we denote by $\tau_{V}$ is the element in $\left(\mathcal{H}^{\prime}\right)^{\widehat{\otimes} 2}$ given by

$$
\left\langle\tau_{V}, z \otimes w\right\rangle=\left\langle z_{V}, w_{V}\right\rangle \quad z, w \in \mathcal{H}_{c} .
$$

The $V$-trace operator can be represented as $\tau_{V}=\sum_{k=1}^{\infty} e_{k} \otimes P_{V} e_{k}$ where $P_{V}$ is the orthogonal projection onto the subspace $V$.

5.7.2. Subspace Wick tensor. With the notion of a subspace trace operator securely behind us, we can now define the subspace Wick tensor. Again we let $V$ be a closed subspace of our Hilbert space $H_{0}$.

Definition 5.10. For $f \in \mathcal{H}^{\prime}$ the $V$-Wick tensor for $f$ of order $n$ is defined to be

$$
: f^{\otimes n}:_{V}=\sum_{k=0}^{[n / 2]}\left(\begin{array}{c}
n \\
2 k
\end{array}\right)(2 k-1) ! !(-1)^{k} f^{\otimes(n-2 k)} \widehat{\otimes} \tau_{V}^{\otimes k} .
$$

Using the notions of the subspace Wick tensor, we are ready to present the Wiener-Itô expansion of $\tilde{\delta}_{a+V}$.

Theorem 5.11. The Wiener-Itô expansion of $\tilde{\delta}_{a+V}$ is given by

$$
\sum_{n=0}^{\infty} \frac{1}{n !} I_{n}\left(: a^{\otimes n}:_{V^{\perp}}\right) .
$$

The proof of this would take us far off course. The interested reader can refer to 2 . 
Remark 5.12. The case we will be primarily interested in for the algorithm happens when $a=0$. Combining Theorem 5.11 along with Definition 5.10, we have the elements of the expansion for $\tilde{\delta}_{V}$ are given by

$$
\begin{cases}(-1)^{n / 2} \frac{(n-1) ! !}{n !} \tau_{V^{\perp}}^{n / 2} & n \text { even, } \\ 0 & n \text { odd. }\end{cases}
$$

\section{Results involving $\mu_{V}$}

In this section we provide the white noise analogs of identities which are needed in the Hidden Subspace algorithm. The first is an infinite dimensional analog of the finite dimensional equation given by

$$
\int_{\mathbb{R}^{n}} f(x) d x=\int_{V} \int_{V^{\perp}} f(v+w) d v d w
$$

where $V$ is a subspace of $\mathbb{R}^{n}$ and $f$ is a suitable function.

The second identity is a generalization of (2.1). This is an analog of the finite dimensional identity

$$
\int_{V} f(x) d x=\int_{V^{\perp}} \hat{f}(k) d k
$$

where $\hat{f}$ is the Fourier transform of a regular enough function $f$ on $\mathbb{R}^{n}$, and $V$ is a subspace of $\mathbb{R}^{n}$. This finite dimensional identity appears in Hörmander [5] (Theorem 7.1.25), where it is generalized to submanifolds of $\mathbb{R}^{n}$ and several consequences developed.

6.1. Decomposition theorem. Using this framework of the Hida measure $\mu_{a+V}$ and the corresponding distribution $\tilde{\delta}_{a+V}$, we have the following intuitive theorem:

Theorem 6.1. Let $V$ be a closed subspace of $H_{0}$, then for any $\phi \in[\mathcal{H}]$ we have

$$
\int_{\mathcal{H}^{\prime}} \phi(x) d \mu(x)=\int_{\mathcal{H}^{\prime}} \int_{\mathcal{H}^{\prime}} \phi(x+y) d \mu_{V}(x) d \mu_{V \perp}(y) .
$$

Proof. To begin we let $\phi=e^{i \hat{u}}=e^{i\langle\cdot, u\rangle}$ for some $u \in \mathcal{H}$. Then on the left we have that

$$
\int_{\mathcal{H}^{\prime}} e^{i \hat{u}} d \mu(x)=e^{-\frac{1}{2}|u|_{0}^{2}}
$$

and on the right we have

$$
\begin{aligned}
\int_{\mathcal{H}^{\prime}} \int_{\mathcal{H}^{\prime}} e^{i\langle u,(x+y)\rangle} d \mu_{V}(x) d \mu_{V^{\perp}}(y) & =\int_{\mathcal{H}^{\prime}} \int_{\mathcal{H}^{\prime}} e^{i\langle u, y\rangle} e^{i\langle u, x\rangle} d \mu_{V}(x) d \mu_{V^{\perp}}(y) \\
& =\int_{\mathcal{H}^{\prime}} e^{i\langle u, y\rangle} \int_{\mathcal{H}^{\prime}} e^{i\langle u, x\rangle} d \mu_{V}(x) d \mu_{V^{\perp}}(y) \\
& =e^{-\frac{1}{2}\left|u_{V}\right|_{0}^{2}} e^{-\frac{1}{2}\left|u_{V} \perp\right|_{0}^{2}} \\
& =e^{-\frac{1}{2}|u|_{0}^{2}}
\end{aligned}
$$

Thus the left and right sides agree on the linear span of $\left\{e^{i \hat{u}} ; u \in \mathcal{H}\right\}$. 
Let $\phi$ be an arbitrary element of $[\mathcal{H}]$. Using Theorem 4.3 , let $\phi_{n}$ be a sequence in the linear space of $\left\{e^{i\langle\cdot, u\rangle} ; u \in \mathcal{H}\right\}$ such that $\phi_{n}$ converges to $\phi$ in $[\mathcal{H}]$. Then we have that

$$
\begin{aligned}
\int_{\mathcal{H}^{\prime}} \phi(x) d \mu(x) & =\langle\langle\phi, 1\rangle\rangle \\
& =\lim _{n \rightarrow \infty}\left\langle\left\langle\phi_{n}, 1\right\rangle\right\rangle \\
& =\lim _{n \rightarrow \infty} \int_{\mathcal{H}^{\prime}} \phi_{n}(x) d \mu(x) \\
& =\lim _{n \rightarrow \infty} \int_{\mathcal{H}^{\prime}} \int_{\mathcal{H}^{\prime}} \phi_{n}(x+y) d \mu_{V}(x) d \mu_{V^{\perp}}(y) .
\end{aligned}
$$

Now we must bring the limit inside both integrals. We first apply Theorem 5.5 to $\mu_{V} \perp$ to see that the measure is supported on $H_{k}^{\prime}$ for some $k$. Hence the above is

$$
=\lim _{n \rightarrow \infty} \int_{H_{k}^{\prime}} \int_{\mathcal{H}^{\prime}} \phi_{n}(x+y) d \mu_{V}(x) d \mu_{V^{\perp}}(y) .
$$

Using the translation operator $T_{y}$, the above becomes

$$
\begin{aligned}
& =\lim _{n \rightarrow \infty} \int_{H_{k}^{\prime}} \int_{\mathcal{H}^{\prime}} T_{y} \phi_{n}(x) d \mu_{V}(x) d \mu_{V^{\perp}}(y) \\
& =\lim _{n \rightarrow \infty} \int_{H_{k}^{\prime}}\left\langle\left\langle T_{y} \phi_{n}, \tilde{\delta}_{V}\right\rangle\right\rangle d \mu_{V^{\perp}}(y) .
\end{aligned}
$$

We would like to apply the dominated convergence theorem to (6.2). To do so take $p \geq k$ such that $\left\|\tilde{\delta}_{V}\right\|_{-p}<\infty$ and let $f_{n}(y)=\left\langle\left\langle T_{y} \phi_{n}, \tilde{\delta}_{V}\right\rangle\right\rangle$. Note that

$$
\begin{aligned}
\left|f_{n}(y)\right| & =\left|\left\langle\left\langle T_{y} \phi, \tilde{\delta}_{V}\right\rangle\right\rangle\right| \\
& \leq\left\|T_{y} \phi_{n}\right\|_{p}\left\|\tilde{\delta}_{V}\right\|_{-p} .
\end{aligned}
$$

We now take $q>p$ satisfying $\lambda_{1}^{2(q-p)}>2$ in order to apply Theorem 4.8 to see that the above

$$
\leq\left\|\tilde{\delta}_{V}\right\|_{-q}\left\|\phi_{n}\right\|_{q}\left(1-\frac{2}{\lambda_{1}^{2(q-p)}}\right) \exp \left[\frac{1}{\lambda_{1}^{2(q-p)}}|y|_{-p}^{2}\right]
$$

Now since $\phi_{n}$ converges to $\phi$ we have that $\left\|\phi_{n}\right\|_{q}$ is bounded. Also using that $\lambda_{1}^{2(q-p)} \geq 2$, we have

$$
\left|f_{n}(y)\right|=\left|\left\langle\left\langle T_{y} \phi, \tilde{\delta}_{V}\right\rangle\right\rangle\right| \leq K e^{\frac{1}{2}|y|_{-p}^{2}} .
$$

Because $p \geq k$ and $k$ was chosen to satisfy Theorem 5.5. we have that the $K e^{\frac{1}{2}|y|_{-p}^{2}}$ is an integrable function with respect to $\mu_{V^{\perp}}$. Then we can apply the dominated convergence theorem to (6.2) to get

$$
\int_{\mathcal{H}^{\prime}} \phi(x) d \mu(x)=\int_{H_{k}^{\prime}} \lim _{n \rightarrow \infty}\left\langle\left\langle T_{y} \phi_{n}, \tilde{\delta}_{V}\right\rangle\right\rangle d \mu_{V^{\perp}}(y) .
$$


Using Theorem 4.8, we have that the translation operator is continuous. Therefore $\lim _{n \rightarrow \infty}\left\langle\left\langle T_{y} \phi_{n}, \tilde{\delta}_{V}\right\rangle\right\rangle=\left\langle\left\langle T_{y} \phi, \tilde{\delta}_{V}\right\rangle\right\rangle$. Hence the above becomes

$$
\int_{\mathcal{H}^{\prime}} \phi(x) d \mu(x)=\int_{H_{k}^{\prime}}\left\langle\left\langle T_{y} \phi, \tilde{\delta}_{V}\right\rangle\right\rangle d \mu_{V^{\perp}}(y) .
$$

Converting back to the measure $\mu_{V}$ we have the result:

$$
\int_{\mathcal{H}^{\prime}} \phi(x) d \mu(x)=\int_{\mathcal{H}^{\prime}} \int_{\mathcal{H}^{\prime}} \phi(x+y) d \mu_{V}(x) d \mu_{V^{\perp}}(y) .
$$

6.2. Integral identity. The integral identity found in (2.1) plays a large role in the Hidden Subspace Algorithm outlined in Section 2. Here we prove the white noise generalization of the identity. This identity was originally developed and proved in [1]. We repeat the proof here in the interest of completeness.

Theorem 6.2. Let $V$ be a closed subspace of the real, separable, Hilbert space $H_{0}$. Let $\mu_{V}$ be the Gaussian measure for $V$, and let $\mu_{V \perp}$ be the Gaussian measure for $V^{\perp}$, constructed as before. Then, for any test function $\phi \in[\mathcal{H}]$ on $\mathcal{H}^{\prime}$, we have

$$
\int_{\mathcal{H}^{\prime}} \phi d \mu_{V}=\int_{\mathcal{H}^{\prime}} S \phi(i k) d \mu_{V^{\perp}}(k) .
$$

Proof. First consider the function $c_{z}$ for $\phi$, where $c_{z}=e^{\langle\cdot, z\rangle-\frac{1}{2}\langle z, z\rangle}$, where $z \in \mathcal{H}_{c}$. Then the left side of 6.3 is

$$
\int_{\mathcal{H}^{\prime}} c_{z} d \mu_{V}=e^{\frac{1}{2}\left\langle z_{V}, z_{V}\right\rangle} e^{-\frac{1}{2}\langle z, z\rangle}=e^{-\frac{1}{2}\left\langle z_{V^{\perp}}, z_{V^{\perp}}\right\rangle} .
$$

The right side of (6.3) is

$$
\begin{aligned}
\int_{\mathcal{H}^{\prime}} S c_{z}(i y) d \mu_{V^{\perp}}(y) & =\int_{\mathcal{H}^{\prime}} e^{i\langle y, z\rangle} d \mu_{V^{\perp}}(y) \\
& =e^{-\frac{1}{2}\left\langle z_{V^{\perp}}, z_{V^{\perp}}\right\rangle} .
\end{aligned}
$$

Thus our identity (6.3) holds for the functions $c_{z}$, and hence for $\phi$ in the linear span $L$ of these functions. Now take $\phi$ to be any test function in $[\mathcal{H}]$. Then, by Theorem 4.3, there is a sequence of functions $\phi_{n} \in L$ which converges in the topology of $[\mathcal{H}]$ to $\phi$ and hence also pointwise. Moreover, for every $p \geq 0$

$$
\lim _{n \rightarrow \infty} \phi_{n}=\phi \quad \text { in }\|\cdot\|_{p} \text {-norm. }
$$

From the identity

$$
\int_{\mathcal{H}^{\prime}} \phi_{n} d \mu_{V}=\int_{\mathcal{H}^{\prime}} S \phi_{n}(i k) d \mu_{V \perp}(k)
$$

the fact that $\mu_{V}$ is a Hida measure implies that as $n \rightarrow \infty$ the left side converges to $\int_{\mathcal{H}^{\prime}} \phi d \mu_{V}$. On the other hand, using the easily checked $p$-norm for $e^{\langle\cdot, z\rangle-\frac{1}{2}\langle z, z\rangle}$, we have

$$
\left|S \phi_{n}(i k)\right| \leq\left\|\phi_{n}\right\|_{p}\left\|e^{i\langle\cdot, k\rangle+\frac{1}{2}\langle k, k\rangle}\right\|_{-p}=\left\|\phi_{n}\right\|_{p} e^{|k|_{-p}^{2} / 2},
$$

and thus the integrands on the right are bounded by $\left\|\phi_{n}\right\|_{p} e^{|k|_{-p}^{2} / 2}$, for every $p \geq 0$. As noted before $\mu_{V^{\perp}}$ is a Hida measure, and so by Theorem 5.5 there is a $p \geq 1$ for which

$$
\int_{\mathcal{H}^{\prime}} e^{|k|_{-p}^{2} / 2} d \mu_{V^{\perp}}(k)<\infty
$$


Combining these facts we see that the dominated convergence theorem applies to the right side of (6.4) and the latter converges to $\int_{\mathcal{H}^{\prime}} S \phi(i k) d \mu_{V^{\perp}}(k)$. This completes the proof of the identity.

\section{Hidden Subspace Algorithm}

In this section we venture step by step through the algorithm presented in Section 2 As we go, we develop mathematical rigor through use of the white noise setting.

7.1. Original setup. In the original algorithm, we have a functional $\phi: H \rightarrow \mathbb{C}$ with a hidden subspace $V \subset H$. That is, $\phi(x+v)=\phi(x)$ for all $v \in V$.

The original algorithm used the rigged Hilbert spaces $\mathbb{H}$ (see Section 2) and the more common $\left(\mathcal{S}\left(\mathbb{R}^{n}\right), L^{2}\left(\mathbb{R}^{n}\right), \mathcal{S}^{\prime}\left(\mathbb{R}^{n}\right)\right)$, which we abbreviated by $H_{\mathbb{R}^{n}}$.

7.2. White noise setup. Here we have a functional $\phi: \mathcal{H}^{\prime} \rightarrow \mathbb{R}^{n}$ with a hidden subspace $\mathcal{V} \subset \mathcal{H}^{\prime}$. We suppose that $\mathcal{V}$ can be specified by vectors in $H_{0}$. That is, there are vectors $\left\{w_{k}\right\}$ such that $\mathcal{V}=\bigcap_{k} \hat{w}_{k}^{-1}(0)$. The closed subspace of $H_{0}$ consisting of those vectors in the set $\bigcap_{k} \hat{w}_{k}^{-1}(0)$ will be denoted simply by $V$. Again, $\phi(x+v)=\phi(x)$ for all $v \in \mathcal{V}$.

In the white noise setting the two spaces we will use are $\left([\mathcal{H}], L^{2}\left(\mathcal{H}^{\prime}, \mu\right),[\mathcal{H}]^{\prime}\right)$ and $\left(\mathcal{S}\left(\mathbb{R}^{n}\right), L^{2}\left(\mathbb{R}^{n}\right), \mathcal{S}^{\prime}\left(\mathbb{R}^{n}\right)\right)$.

Step 1. In the original algorithm we begin in the state $|0\rangle|0\rangle \in \mathbb{H} \otimes H_{\mathbb{R}^{n}}$. Here, in a similar fashion, we begin in

$$
\tilde{\delta}_{0} \otimes \delta_{0} \text { or } \tilde{\delta}_{0}|0\rangle,
$$

where $\tilde{\delta}_{0}$ is the Kubo-Yokoi delta function and $\delta_{0}$ is the delta function in $\mathcal{S}^{\prime}\left(\mathbb{R}^{n}\right)$.

Step 2. In the original algorithm we applied the operator $\mathcal{F}^{-1} \otimes I$ to create superposition $\int_{H} D x|x\rangle|0\rangle$.

Here we do something similar and apply the Kuo Fourier transform to our previous state. That is we apply $\mathcal{F} \otimes I$ to (7.1) and use that $\mathcal{F} \tilde{\delta}_{0}=1$ (see Example 5.8) to arrive at

$$
1(x)|0\rangle \text {. }
$$

The $x$ is put there to emphasize that 1 is the function which assigns the real number 1 given any $x \in \mathcal{H}^{\prime}$.

Step 3. In the original algorithm we made use of black box $U_{\phi}: \mathbb{H} \otimes H_{\mathbb{R}^{n}} \rightarrow \mathbb{H} \otimes H_{\mathbb{R}^{n}}$ which had the effect of $U_{\phi}|x\rangle|z\rangle=|x\rangle|z+\phi(x)\rangle$. The black box $U_{\phi}$ moves the current state into $\int_{H} D x|x\rangle|\phi(x)\rangle$.

This step is changed the least from the original in that we apply a similar black box to $1(x)|0\rangle$ to arrive at the state

$$
1(x)|\phi(x)\rangle \text {. }
$$

Step 4. Here is where the "meat" of the algorithm takes place. In the original we began in the state $\int_{H} D x|x\rangle|\phi(x)\rangle$ and used the hidden subspace of $\phi$ along with the identity $\int_{V \perp} \delta(y-u) D u=\int_{V} e^{-2 \pi i\langle v, y\rangle} D v$ to arrive at $\int_{V \perp} D u|u\rangle|\Omega(u)\rangle$.

In the white noise version you will notice many similarities. To begin, we use the $\mathcal{T}$-transform and apply the operator $\mathcal{T} \otimes I$ to (17.2). Thus we arrive at the state

$$
\int_{\mathcal{H}^{\prime}} e^{i\langle x, y\rangle} 1(x)|\phi(x)\rangle d \mu(x) \text {. }
$$


Now with regards to the hidden subspace, we use Theorem 6.1 to decompose the above into

$$
\int_{\mathcal{H}^{\prime}} \int_{\mathcal{H}^{\prime}} e^{i\langle v+w, y\rangle}|\phi(v+w)\rangle d \mu_{V}(v) d \mu_{V^{\perp}}(w) .
$$

From Remark 5.1 we know that $\mu_{V}$ lives on $\mathcal{V}$. Thus the above becomes

$$
\int_{\mathcal{H}^{\prime}} \int_{\mathcal{V}} e^{i\langle v+w, y\rangle}|\phi(v+w)\rangle d \mu_{V}(v) d \mu_{V^{\perp}}(w) .
$$

Next we use that $\phi(v+w)=\phi(w)$ for all $v \in \mathcal{V}$ to arrive at

$$
\int_{\mathcal{V}} e^{i\langle v, y\rangle} d \mu_{V}(v) \int_{\mathcal{H}^{\prime}} e^{i\langle w, y\rangle}|\phi(w)\rangle d \mu_{V^{\perp}}(w) .
$$

We now apply the identity found in Theorem 6.2 to arrive at

$$
\int_{\mathcal{H}^{\prime}} e^{\langle u, y\rangle-\langle y, y\rangle / 2} d \mu_{V^{\perp}}(u) \int_{\mathcal{H}^{\prime}} e^{i\langle w, y\rangle}|\phi(w)\rangle d \mu_{V^{\perp}}(w),
$$

or equivalently

$$
S\left(\tilde{\delta}_{V \perp}\right)(y) \int_{\mathcal{H}^{\prime}} e^{i\langle w, y\rangle}|\phi(w)\rangle d \mu_{V \perp}(w) .
$$

Thus we have the $S$-transform of the delta function on the subspace $\tilde{\delta}_{V^{\perp}}$. We can use this to help us identify the hidden subspace.

Step 5. In the original algorithm we measured $\int_{V \perp} D u|u\rangle|\Omega(u)\rangle$ with respect to the observable $M=\int_{H} D w|w\rangle\langle w|$.

For the white noise version we take a different approach which makes use of the rich structure of white noise distribution theory.

For $n=0,1,2, \ldots$, let $P_{n}$ be the orthogonal projection onto the closed linear span of

$$
\left\{\left\langle f_{n},{ }^{\otimes n}\right\rangle ; f_{n} \in \mathcal{H}^{\prime \otimes n}\right\} .
$$

Using the decomposition of $\tilde{\delta}_{V^{\perp}}$ from Remark 5.12, we have see that $P_{n} S\left(\tilde{\delta}_{V^{\perp}}\right)$ will yield 0 if $n$ happens to be odd. More importantly, if $n$ happens to be even, we get $(-1)^{n / 2} \frac{(n-1) ! !}{n !} \tau_{V}^{n / 2}$, a multiple of the trace class operator on $V$.

\section{REFERENCES}

1. J. J. Becnel and A. N. Sengupta, An infinite dimensional integral identity for the SegalBargmann transform, Proceedings of the American Mathematical Society 135 (2007), no. 9, 2995-3003. MR2317978(2008k:60163)

2. Jeremy J. Becnel, Delta function for an affine subspace, Taiwanese Journal of Mathematics 12 (2008), no. 9, 2269-2294. MR2479055(2010a:46094)

3. Jeremy J. Becnel and Ambar N. Sengupta, The Schwartz space: Tools for white noise analysis, preprint at http://www.math.lsu.edu/ preprint/, December 2004.

4. I. M. Gel'fand and N. Ya. Vilenkin, Application of harmonic analysis, Generalized Functions, vol. 4, Academic Press, New York, New York, 1964. MR0435834 (55:8786d)

5. Lars Hörmander, The analysis of linear partial differential operators I, Grundlehren der mathematischen Wissenschaften, Springer-Verlag, Berlin, Heidelberg, 1983. MR717035 (85g:35002a)

6. I. Kubo and Yoshitaka Yokoi, A remark on the space of testing random variable in the white noise calculus, Nagoya Mathematics Journal 115 (1989), 139-149. MR1018088 (91e:60186)

7. H.-H. Kuo, White noise distribution theory, Probability and Stochastic Series, CRC Press, Inc., New York, New York, 1996. MR.1387829 (97m:60056)

8. Hui-Hsiung Kuo, Gaussian measures on banach spaces, Lecture Notes in Mathematics, Vol. 463, Springer-Verlag, Berlin, New York, 1975. MR0461643 (57:1628) 
9. Samuel J. Lomonaco and Louis H. Kauffman, Continuous quantum hidden subgroup algorithms, Proceedings of SPIE, vol. 5105, 2003, pp. 80-88.

10. Nobuaki Obata, White noise calculus and fock space, Lecture Notes in Mathematics, SpringerVerlag, New York, New York, 1994. MR1301775 (96e:60061)

Department of Mathematics and Statistics, Stephen F. Austin State University, NACOGdoches, Texas 75962-3040

E-mail address: becneljj@sfasu.edu

$U R L:$ http://www.faculty.sfasu.edu/becneljj 